\title{
Microdeletions within the hydrophobic core region of cellular prion protein alter its topology and metabolism
}

\author{
Jens Lutz ${ }^{\mathrm{a}, \mathrm{d}}$, Christine Brabeck ${ }^{\mathrm{a}}$, Hartmut H. Niemann ${ }^{\mathrm{b}}$, Ulrich Kloz ${ }^{\mathrm{c}}$, Carsten Korth ${ }^{\mathrm{d}}$, \\ Vishwanath R. Lingappa ${ }^{\mathrm{e}}$, Alexander Bürkle ${ }^{\mathrm{a}, *}$ \\ "Molecular Toxicology Group, Department of Biology, University of Konstanz, Konstana, Gemany \\ Department of Biochemistry. Bielefeld University. Bielefeld, Gemany \\ 'Transgenic Core Facility, Geman Cancer Research Center, Heidelberg, Germany \\ anstitute of Neuropatholosy, Heinich Heine University of Düsseldorf, Gennany

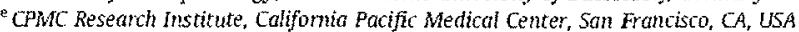

Kenwords:

Prion protein

Transmentrane domain

Membrane topology

$\alpha$-Cleavage

CI fragment

\begin{abstract}
A B S T R A T
The cellular prion protein ( $\mathrm{PrP}^{C}$ ) is a GPI-anchored cell-surface protein. A small subset of PrP molecules, however, can be integrated into the ER-membrane via a transmembrane domain (TM), which also harbors the most highly conserved regions of $\operatorname{Pr} \mathrm{P}^{\mathrm{C}}$, termed the hydrophobic core (HC). A mutation in $\mathrm{HC}$ is associated with prion disease resulting in an enhanced formation of a transmembrane form ( ${ }^{C m} \mathrm{PrP}$ ) which has thus been postulated to be a neurotoxic molecule besides $P_{\mathrm{P}}^{\mathrm{sc}}$. To elucidate a possible phys iological function of the transmembrane domain, we created a set of mutants carrying microdeletions of 2-8 aminoacids within HC between position 114 and 121 . Here, we show that these mutations display reduced propensity for transmembrane topology. In addition, the mutants exhibited alterations in the formation of the $\mathrm{C} 1$ proteolytic fragment, which is generated by $\alpha$-cleavage during normal $\operatorname{PrP}^{\mathrm{C}}$ metab olism, indicating that $\mathrm{HC}$ might function as recognition site for the protease(s) responsible for $\operatorname{PrPC}^{C} \alpha-$ cleavage. Interestingly, the mutant G113V, corresponding to a hereditary form of prion disease in humans, displayed increased ${ }^{c m p}$ prp topology and decreased $\alpha$-cleavage in our in vitro assay. In conclusion, $\mathrm{HC}$ represents an essential determinant for transmembrane PrP topology, whereas the high evolutionary conservation of this region is rather based upon preservation of $\operatorname{Pr}^{C} \alpha$-cleavage, thus highlighting the biological importance of this cleavage.
\end{abstract}

\section{Introduction}

Prions are the causative agents of transmissible spongiform encephalopathies (TSEs). These fatal neurodegenerative diseases, including BSE in cattle, Scrapie in sheep and Creutzfeldt-jakob disease (CJD) in humans, can be of infectious or genetic etiology or

Abbreviations: $\alpha 1-3, \alpha$-helical structure; $\beta 1-2, \beta$-sheet structure; aa, aminoacids; $A b$, antibody; ADAM, $A$ disincegrin and metalloproteinase; $A P$, acceptor peptide; APP, amyloid precursor protein; $\mathrm{Cl}, \mathrm{C}$-teminal fragment of PrP after $\alpha$ cleavage; $\mathrm{Cu}$, $\mathrm{C}$ pper binding sites; $\mathrm{C} A \mathrm{~A}$, antibody directed against the $\mathrm{C}$-terminus of $\mathrm{PrP}$; ${ }^{\mathrm{C} r \mathrm{P}} \mathrm{PrP}, \mathrm{C}$-terminal transmemorane topology of $\mathrm{PrP} ; \mathrm{HC}$, hydrophobic core; GPI, glycosyl-phosphatidylinositol anchor signal sequence; IP, immunoprecipitation; Mem, microsomal membranes; MoPrP, mouse PrP; $\mathrm{N} 1, \mathrm{~N}$-terminal fragment of PrP after $\alpha$-cleavage; $N$-Ab, antibody, directed against the N-terninus of PrP. NeraprP, N-terminal transmembrane topology of PrP; Octa-R, octa-repeats: PK, proteinase $\mathrm{K}$; $\mathrm{Pr} \mathrm{PC}^{\mathrm{C}}$, cellular, physiological prion protein; $\mathrm{SS}$, signal sequence; $T M$. transmembrane (donain); wh, wild-type.

* Corresponding author. Address: Department of Biology, Box X911, University of Konstanz, 78457 Konstanz, Germany. Fax: +497531884033.

E-mail address: alexander.buerkle@uni-konstanzde (A Bürkle). arise sporadically. They are characterized by the conversion of $\operatorname{Pr} \mathrm{P}^{\mathrm{C}}$ into a disease-associated isoform ( $\mathrm{Pr}^{\mathrm{Sc}}$ ), which shows resistance to proteinase $\mathrm{K}(\mathrm{PK})[1]$.

$\operatorname{PrPC}^{\mathrm{C}}$ is mainly expressed in neurons and is attached to the outer surface of the cell membrane via a C-terminal glycosyl-phosphatidylinositol (GPI) anchor. In addition to the cell-surface anchored version of $\mathrm{Pr} \mathrm{PC}^{\mathrm{C}}$, termed ${ }^{\text {sec }} \mathrm{PrP}$, transmembrane topologies with either the $\mathrm{N}$-terminus ( ${ }^{\mathrm{N} t \mathrm{~m}} \mathrm{PrP}$ ) or the $\mathrm{C}$-terminus ( ${ }^{\mathrm{Cm}} \mathrm{Pr} \mathrm{P}$ ) facing the lumen of the endoplasmic reticulum (ER) have been described [2]. These are inserted into the ER-membrane via the TM of PrPC, a hydrophobic stretch of aminoacids 111-134 encompassing the highly conserved HC (residues 112-119). The mutation A117V is associated with heritable prion disease and displays increased proportion of ${ }^{\mathrm{Cm}} \mathrm{PrP}$ topology. It causes spontaneous neurodegeneration in transgenic mice, but brain tissue of the affected animals lacks infectivity. It has thus been hypothesized that Ctraprp represents the neurotoxic species during prion infection $[2,3]$. The importance of the TM region for disease is reflected by the fact that a peptide encompassing aminoacids 105-125 has widely been 
used as a neurotoxic model peptide [4,5]. Furthermore, various interaction partners such as stress-inducible protein 1 (STl-1) and also $\mathrm{PrP}^{\mathrm{Sc}}$ bind to TM [6,7]. The latter binding is essential for the conversion of $\operatorname{PrP}^{C}$ into $\operatorname{PrP}^{5 C}[8]$. This is exemplified by $\operatorname{PrP}$ mutants with deletion of the aminoacids 114-121 or 112-119 [7,9].

During its normal metabolism, mature $\mathrm{PrPC}^{\mathrm{C}} \mathrm{can}$ be cleaved at two sites, i.e. $\beta$-cleavage at around residue 90 or $\alpha$-cleavage at position 109-111, resulting in small $\mathrm{N}$-terminal (N1 and N2, respectively) and larger $\mathrm{C}$-terminal fragments ( $\mathrm{C} 1$ and $\mathrm{C2}$ ) [10]. Such cleavage reactions are reminiscent of cleavage events in the Alzheimer amyloid precursor protein (APP) [11].

Here we constructed a set of microdeletions within $\mathrm{TM}$ of mouse $\operatorname{PrP}(\mathrm{MoPrP})$ to investigate the role of $\mathrm{TM}$ in $\mathrm{PrP}^{\mathrm{C}}$ topology. Our data reveal that these deletions lead to alterations both in membrane topology and in $\alpha$-cleavage.

\section{Materials and methods}

Unless stated otherwise, all aminoacid numbering refers with the murine PrP sequence. All chemicals were purchased from Sigma-Aldrich, Germany.

Plasmids. Plasmid pUC19::MoPrP-wt [9], comprising the coding sequence for MoPrP, was used to generate a set of eight deletion mutants. For in vitro topology assays, the various coding sequences were cloned into pTNT vector (Promega) containing the SP6 promoter. The G113V mutation was created by mutagenesis of pTNT::MoPrP-wt using the Quick change site directed mutagenesis kit (Stratagene) by using the primers G113V-forward ( $5^{\prime}$ CAACCT CAAGCATGTGGCAGTGGCCGCGGCAGCTGGGGCAGTAG 3') and G113V-reverse (5' CTACTGCCCCAGCTGCCGCGGCCACTGCCACATGC TTGAGGTTG $3^{\prime}$ ).

Transgenic mice. The generation of the $\mathrm{F} 902$ line of transgenic mice expressing MoPrP-1 $14 \Delta 8$ has been described [12]. In parallel another transgenic line termed M630 was created. Offspring of the two lines were backcrossed four times on a Pmp $p^{-1-}$ background by mating with Prnp ${ }^{-1-}$ FVB mice.

In vitro topology assay and determination of $\alpha$-cleavage in vivo. Transcription and translation of the various PrP mutants was carried out essentially as described [2]. A detailed description is provided under Supplementary Data.

Statistical analysis and similarity calculations. For each PrP-construct, the proportion of any $\operatorname{PrPC}^{\mathrm{C}}$ fragment was calculated by dividing its band intensity, obtained by densitometry, by the sum of the band intensities of all fragments generated. The difference in the proportion between PrP-wt and the deletion mutant was then analyzed by One-way ANOVA in combination with Tukey's multiple comparison test. Two groups were compared by Student's t-test. All statistical analyses were performed using Prism GraphPad software. The similarity of HC from aminoacid 114-119 between PrP-wt and the different deletion mutants was assessed by assigning to each aminoacid a value depending on its similarity to the original aminoacid in the wt sequence, using the BLOSUM100 matrix [13]. The higher this value, the more similar is the character of the sequence at this position to PrP-wt. An identical alanine for example, is given the value 8 , whereas its substitution to glycine changes this value to -1 , and with lysine to -4 . The sum of the respective values ( = similarity index) is a measure of the similarity of the character of the respective residues $114-119$ to the wt sequence. The correlation of transmembrane forms and $\alpha-$ cleavage with the different deletions of the TM was assessed using linear regression.

\section{Results}

Characterization of $P_{r} P^{C}$ polypeptides generated in the in vitro topology assay

During cellular trafficking and protein maturation, subpopulations of PrP insert into the membrane of the ER via TM. Using a set of mutants bearing microdeletions within its $\mathrm{HC}$ region (Fig. 1), we asked whether these deletions would affect the ability of $\mathrm{PrP}^{\mathrm{C}}$ to adopt transmembrane topology. We therefore, translated the deletion mutants in vitro in the presence of microsomal membranes, followed by PK digestion in the absence of detergents. This leads to digestion of the cytosolic protein parts and thus truncation of the transmembrane forms of $\mathrm{PrP}^{\mathrm{C}}$, either at the N-terminus (for ${ }^{C} \mathrm{PrP}$ ) or at the $\mathrm{C}$-terminus (for ${ }^{\mathrm{N} t m} \mathrm{PrP}$ ), resulting in a distinct fragment size for each topological conformation [2].

First, we confirmed the identity and further characterized the properties of the PrP fragments from topology assays by immunoprecipitation with antibodies either directed against the $\mathrm{N}$ - or the $\mathrm{C}$-terminus of $\operatorname{PrP}^{\mathrm{C}}$ (Supplementary Fig. 1). As expected, an N-terminal antibody precipitated the normal full-length ${ }^{\sec } \mathrm{Pr}$ ( tory $\mathrm{PrP}$ ) as well as the transmembrane fragment NemPrP. Fragments that comprise an intact $C$-terminal region, such as ${ }^{\mathrm{sec}} \mathrm{Pr} P$ and ${ }^{C t m}$ PrP, on the other hand, were immunoprecipitated with the

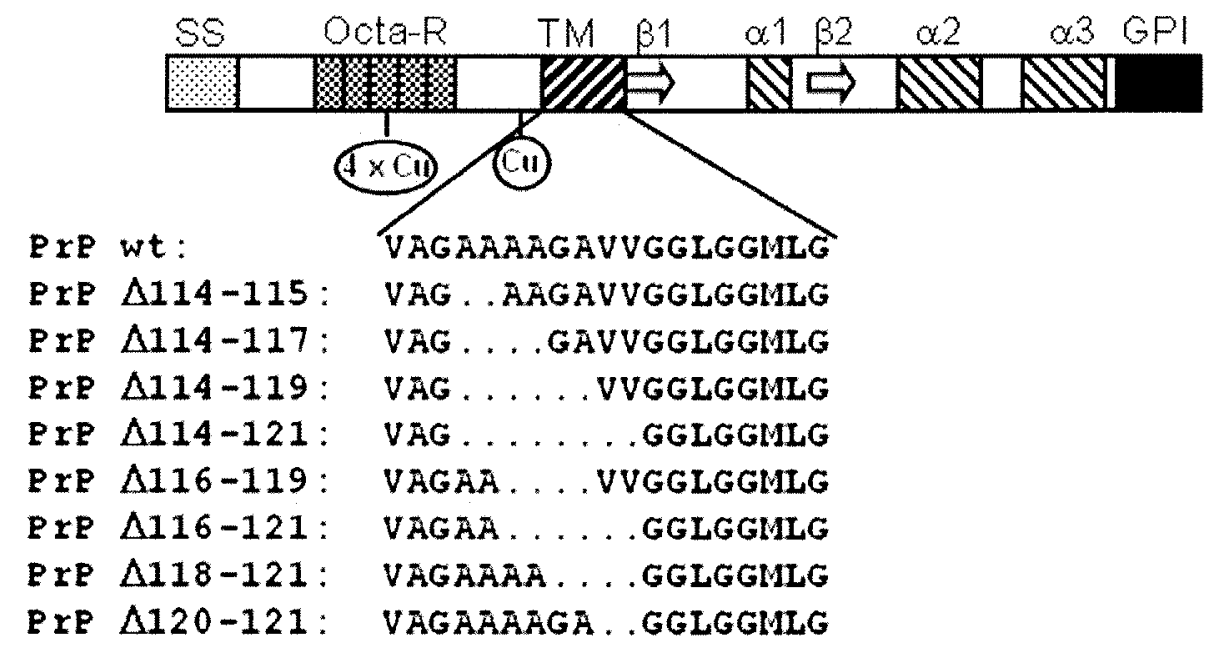

Fig. 1. Mouse PrP deletion mutants. Schematic drawing of mouse PrPC. Underneath the scheme, the aminoacid sequence displays the deletions within HC for each mutant. Q1-3: $\alpha$-helix; $\beta 1-2: \beta$-sheet; Cu: Copper binding sites; GPI-anchor signal sequence; Octa-R: octa-repeats; SS: signal sequence; TM: transmembrane domain. 
C-terminal antibody $6 \mathrm{H} 4$. In addition to these expected fragments, immunoprecipitation with the $6 \mathrm{H} 4$ revealed a fragment of $17-$ $18 \mathrm{kDa}$. This $\mathrm{N}$-terminally truncated fragment already appeared in the absence of PK (Supplementary Fig. 1A, lane 1) and was therefore not generated by PK digestion. Size and truncation rather corresponded to the $\mathrm{C} 1$ fragment created by $\alpha$-cleavage during metabolism of $\operatorname{PrP}^{\mathrm{C}}[10]$. The deletion mutant PrPA114-121 displayed almost complete loss of CtmprP and NtmPrP topologies as well as of $\mathrm{Cl}$ (Supplementary Fig. 1).

\section{The $18 \mathrm{kDa}$ fragment lacks the $3 F 4$ epitope}

In the original paper describing the $\mathrm{C} 1$ fragment [10], $\mathrm{C} 1$ was identified by the lack of immunostaining with antibody $3 \mathrm{~F} 4$ recognizing residues $109-112$ in human and hamster brain as this epitope overlaps with the $\alpha$-cleavage site. Since $3 F 4$ does not recognize MoPrP, we analyzed hamster PrP-wt in the topology assay, immunoprecipitating the fragments generated after PK digestion either with 3F4 or with a C-terminal antibody $6 \mathrm{H} 4$ (Supplementary Fig. 2). Whereas the 18-kDa band was visible as one of the fragments precipitated with $6 \mathrm{H} 4$, the antibody $3 \mathrm{~F} 4$ was unable to precipitate this fragment. This observation further substantiates that the C-terminal $18-\mathrm{kDa}$ fragment is $\mathrm{C} 1$ as originally defined by Autilio-Gambetti and colleagues by the loss of the 3F4 epitope after $\operatorname{Pr} \alpha \alpha$-cleavage.

\section{Deletions in the hydrophobic core region of $\mathrm{Pr}^{\mathrm{C}}$ influence} transmembrane topology and $\alpha$-cleavage

To study the influence of $\mathrm{HC}$ on $\mathrm{PrP}^{\mathrm{C}}$ membrane topology and $\alpha$ cleavage in more detail, the deletion mutants (Fig. 1) were analyzed in the topology assay and most showed a significant decrease in transmembrane topology and also marked decrease in $\alpha$-cleavage as demonstrated by decreased $\mathrm{C} 1$ levels (Supplementary Fig. 3; Fig. 2).

Deletion of aminoacids $114-121$ causes the loss of $\alpha$-cleavage in vivo

The effect of HC on $\mathrm{Pr}^{C} \alpha$-cleavage is also present in vivo. We have created two lines of transgenic mice expressing the deletion mutant PrPA114-121 on a Pmp ${ }^{-1-}$ background, one of which (F902) has been published [12]. While an 18-kDa band was clearly detectable in non-transgenic Prnp ${ }^{+/ *}$ mouse tissue using antibody $6 \mathrm{H} 4$ (Fig. 3A), the intensity of this fragment was dramatically reduced in both transgenic mouse lines expressing PrP $\triangle 114-121$.

In order to clearly demonstrate that the $18 \mathrm{kDa}$ band was indeed $\mathrm{C} 1$, we re-probed the blot with an antibody directed against the $\mathrm{N}$-terminus of $\mathrm{PrP}^{\mathrm{C}}$. The lower band was no longer present in the PrP-wt samples, confirming the identity of the 18-kDa band as the $\mathrm{C} 1$ fragment (Fig. $3 \mathrm{C}$ ).

In both transgenic lines, the level of PrPA114-121 expression was markedly reduced compared to $\mathrm{PrP}^{\mathrm{C}}$ in wt-mice [12] (Fig. 3A). To rule out that this low level of mutant protein expression caused the respective $\mathrm{C} 1$ band to be below the level of detection threshold, the blot was overexposed, resulting in a faint $\mathrm{C} 1$ band (Fig. $3 \mathrm{~B}$ ). Thus, the reduction of the $\mathrm{C} 1$-fragment in the transgenic mice is an authentic result and not an artifact due to the limited quantity of $\operatorname{PrP}$ loaded on the gel.

The data from the densitometric analysis of the Western blot were consistent with the in vitro topology results (Fig. 2). In both experimental setups, PrP $\Delta 114-121$ displayed a near-complete loss of $\alpha$-cleavage. The residual $C 1$ levels in vivo were $2.0 \%$ in transgenic line $\mathrm{M} 630$ and $3.4 \%$ in line $\mathrm{F} 902$; in vitro the residual $\mathrm{C} 1$ level was $1.5 \%$ (Fig. 3D).

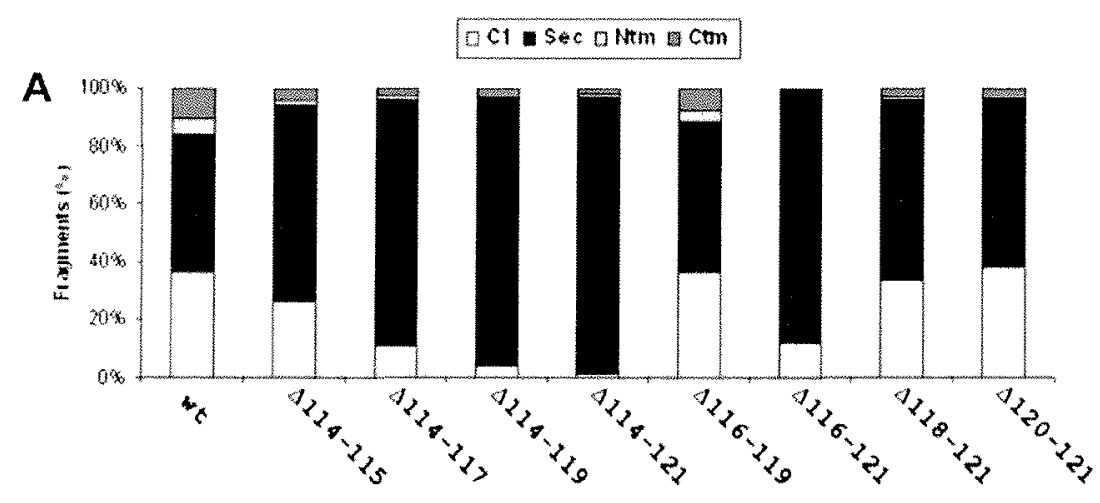

\begin{tabular}{|c|c|c|c|c|c|c|c|c|c|}
\hline$B$ & Wt & $\Delta 114-115$ & $\Delta 114-117$ & $\Delta 114-119$ & $\Delta 114-121$ & $\Delta 116-119$ & $\Delta 116-121$ & $\Delta 118-121$ & $\Delta 120-121$ \\
\hline Ctm & $1 \angle 1 \angle 1$ & $\star \star \star *$ & $* * *$ & $* * *$ & *** & * & $* * *$ & $* \star *$ & $\star * \star *$ \\
\hline Ntm & & $* *$ & ** & $* * *$ & $* \star *$ & n.s. & $* * *$ & $* * *$ & $\star \star \star *$ \\
\hline Sec & & $\star * *$ & $\star * * *$ & $* * *$ & $* * *$ & n.s. & $* * *$ & ** & n.s. \\
\hline $\mathrm{C1}$ & $1 / 1 / 1$ & ** & $\star * *$ & $* * *$ & $* * *$ & n.s. & $* * *$ & n.s. & n.s. \\
\hline
\end{tabular}

Fig. 2. Quantification of topology assessment of the different PrP deletion mutants. (A) Quantification of the ratios of each topological form (black: full-length Ser PrP; light grey: "NmPrP; dark grey: CimprP) and the $C 1$-fragment (white) for each PrPs mutant. Signal intensities of the different fragments were quantifed by densitometry, $n>3$ independent experiments. (B) Statistical significance of the differences in fragment abundance for each mutant conpared to PrP-wt was calculated using one-way ANOVA/ Tukey's multiple comparison test (* denotes $p<0.05 ;^{* *} p<0.01 ;{ }^{* * *} p<0.001 ;$ n.s., not significant). 


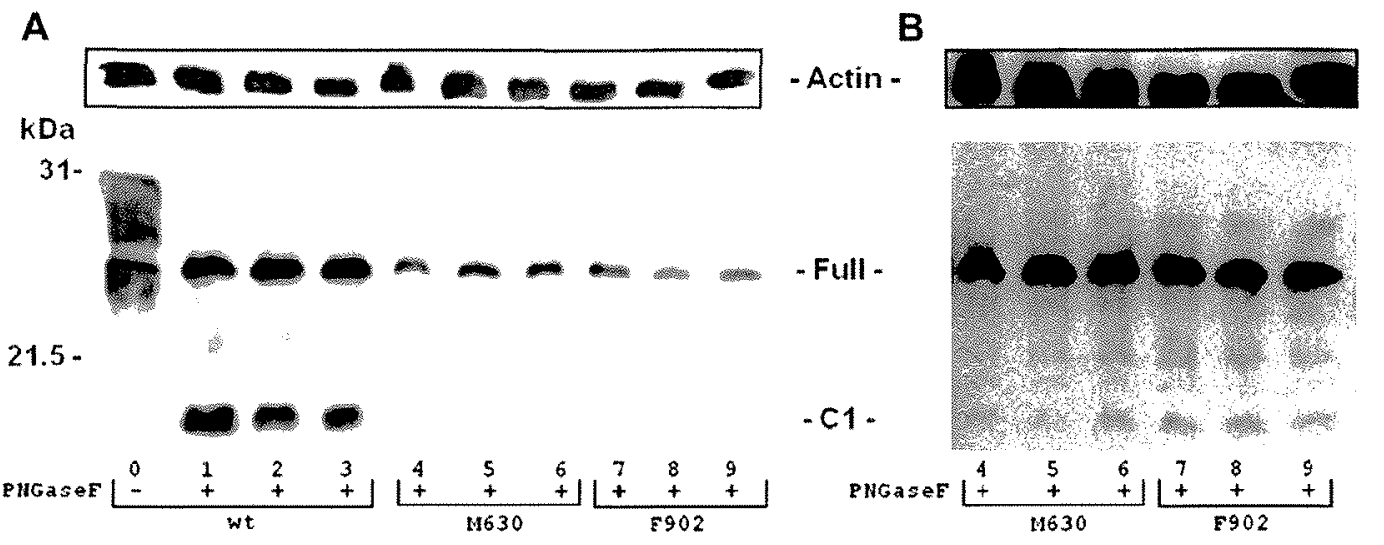

C KDa

D

C1 - Full
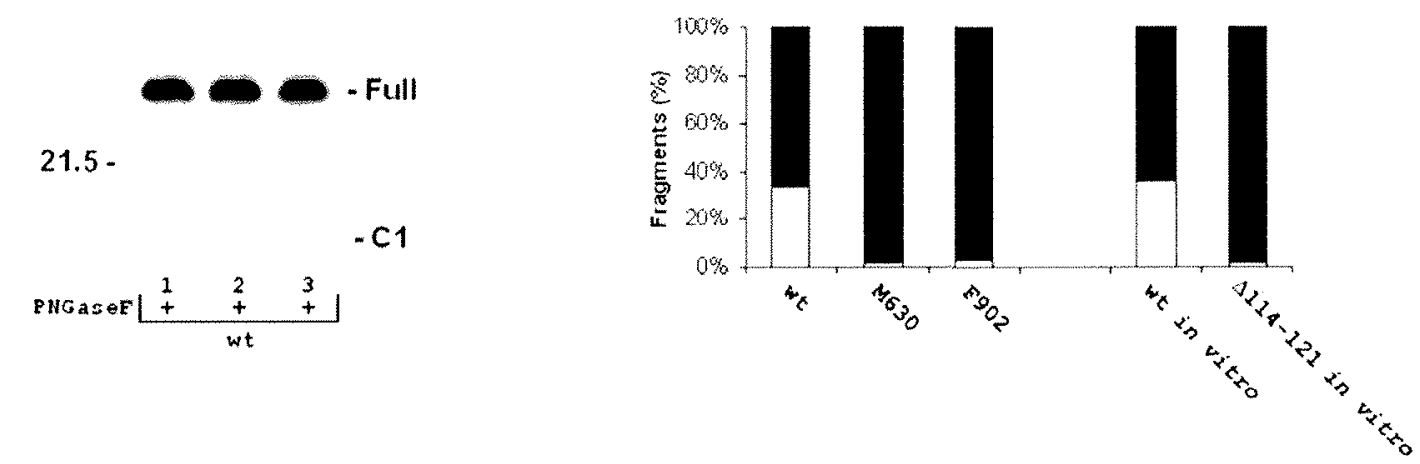

Fig. 3. Pr $\mathrm{P}^{\mathrm{C}} \alpha$-cleavage analysis in vivo. (A) Western blot analysis of brain homogenates from PrP-wt (lanes Q-3) and transgenic mice expressing PrP $\Delta 114-121$ on a Pmp background (M630; lanes 4-6) and (F902; lanes 7-9). For each genotype, equal anounts of brain homogenates from 3 mice were either deglycosylated by PNGaseF (lanes 19) or left untreated as a control ( $\mathrm{PrP}$-Wt; lane 0). Actin was used as a loading control. Non-cleaved full-length PrPC "Full") and the C1 fragment were visualized by probing the blot with antibody $6 \mathrm{H} 4$. A strong $\mathrm{C} 1$ band was only present in the PrP-wt samples. (B) Section showing the mutant samples from the blot shown in panel A. In this overexposed blot, very low levels of $C 1$ indeed become visible in brains from mice expressing PrP $\Delta 114-121$. (C) Section of the PrP-wr samples from the blot of panel (A). After removal of the $\mathrm{C}$-terminal antibody $6 \mathrm{H} 4$, the blot was re-probed with an $\mathrm{N}$-terminal antibody. Only full-fength PrPC was visible. After cleavage, the resulting $\mathrm{C} 1$ fragment has lost the epitope for the $\mathrm{N}$-terminally binding antibody. This result further validates the identity of the lower band as $\mathrm{Cl}$. (D) Quantification of the PrPC fragments in vivo. Signal intensities in panel (A) were quantified by densitometry. The two bars on the tight-hand side indicate the anount of $C 1$ versus non-cleaved fragments (sec PrP plus the transmembrane forms Nin PrP and Ctrn FrP) from the in vitro topology assay (see also Fig. 2). The in vitro and in vivo results showed a comparable decrease in $\alpha$-cleavage for PrPA114-121 (in wivo: $2.0 \%$ in transgenic line M630, 3.48 in line F902; in vitro: $1.5 \%$ ) compared to $33.7 \% \mathrm{Cl}$ for PrP-wt in vivo and $33.6 \%$ in vitro.

Alterations in $\mathrm{Pr}^{\mathrm{P}} \alpha$-cleavage correlate with the aminoacid sequence of $\mathrm{HC}$

The extent of loss of $\operatorname{PrP}^{C} \alpha$-cleavage in the different mutants is not simply a function of deletion size. The four-aminoacid deletion in PrPA114-117, for example, reduced the level of $\mathrm{C} 1$ to $30 \%$, whereas a different four-aminoacid deletion in PrPA116-119 had no effect on $\alpha$-cleavage. Based on this striking observation we systematically compared sequence similarity between the mutants and PrP-wt between positions 114 and 119 (Fig. 4) and observed that the efficiency of $\operatorname{PrP} \alpha$-cleavage is directly correlated with the similarity of this segment to the wt sequence. The last two aminoacids of the deletion region, i.e residues 120-121, have no influence on cleavage efficiency, demonstrated by unchanged $\alpha-$ cleavage efficiency in PrP $\Delta 120-121$. Furthermore, the comparison of segments 114-120 or 114-121 between mutants and wt exhibited a much weaker correlation than the comparison of segment 114-119 (data not shown).

The amount of transmembrane topology and $\alpha$-cleavage is altered in a pathologic mutation of PrP

Two hereditary forms of human prion diseases are associated with missense mutations in $\mathrm{HC}$, ie. G114V and A117V [14]. It is known that $\mathrm{A} 117 \mathrm{~V}$ is associated with an increase in ${ }^{\mathrm{Ctm}} \mathrm{PrP}[2]$, suggesting that the pathologic impact of mutations within HC could be associated with alterations in the metabolism of $\mathrm{PrPC}^{\mathrm{C}}$, such as the upregulation of a toxic transmembrane PrP form, i.e. CtnPrP. We asked whether G114V (corresponding to G113V in MoPrP) may also impact physiologic $\mathrm{Pr}^{\mathrm{C}}$ metabolism. Our in vitro topology assay indeed revealed a significant, threefold increase of crmprp topology from $10.5 \%$ to $32.7 \%$, as well as a decrease in $\alpha$-cleavage by $50 \%$ (Supplementary Fig. 4 ).

\section{Discussion}

HC supports the integration but not the orientation of $\operatorname{Pr} \mathrm{P}^{C}$ into the lipid bilayer

We have created a series of mutants bearing microdeletions within the most bighly conserved region of $\operatorname{Pr} \mathrm{P}^{\mathrm{C}}$, i.e. the $\mathrm{HC}$ region. We could demonstrate that these mutants are inserted less effciently into microsomal membranes, thereby leading to a pronounced reduction of the transmembrane $\mathrm{PrP}^{\mathrm{C}}$ forms ${ }^{\mathrm{Ctm}} \mathrm{PrP}$ and ${ }^{M}{ }^{2} P r P$. This decrease demonstrates that also the $N$-terminal part of TM, ie the hydrophobic core, plays a considerable role in transmembrane topology. This region is necessary for membrane integration of both ${ }^{\text {stm }}$ PrP and ctmprP. The pronounced effect of these 


\begin{tabular}{|c|c|c|c|c|c|c|c|c|c|c|c|}
\hline & & & & no ac & a posi & jon & & & Similarity & TM fragments $(\%)$ & C1 fragment $(\%)$ \\
\hline & 114 & 115 & 116 & 117 & 118 & 119 & 120 & 121 & $114-119$ & & \\
\hline wt & $A$ & A & A & A & $\mathbf{G}$ & A & V & V & 49 & $16 \pm 2$ & $37 \pm 7$ \\
\hline$\Delta 114-115$ & $A$ & A & $G$ & A & v & $v$ & $G$ & $G$ & 13 & $6 \pm 2$ & $26 \pm 5$ \\
\hline$\Delta 114-117$ & G & A & $v$ & v & G & $G$ & $L$ & $G$ & 11 & $4.0 \pm 2$ & $11 \pm 5$ \\
\hline$\Delta 114-119$ & $v$ & $v$ & $G$ & $G$ & $L$ & $G$ & $G$ & $Y$ & -10 & $4 \pm 1$ & $4 \pm 1$ \\
\hline$\Delta 114-121$ & G & G & L & $\mathrm{G}$ & $\mathbf{G}$ & $Y$ & $M$ & $\mathrm{~L}$ & -3 & $3 \pm 2$ & $2 \pm 1$ \\
\hline$\Delta 116-119$ & $A$ & A & $v$ & $v$ & $\mathbf{G}$ & $G$ & $\mathrm{~L}$ & $G$ & 20 & $11 \pm 2$ & $36 \pm 4$ \\
\hline$\Delta 116-121$ & A & A & $G$ & $G$ & $L$ & $\mathrm{G}$ & $G$ & Y & 5 & $1 \pm 1$ & $12 \pm 5$ \\
\hline$\Delta 118-121$ & A & A & $A$ & $A$ & G & $G$ & $L$ & G & 40 & $4 \pm 1$ & $33 \pm 5$ \\
\hline$\Delta 120-121$ & A & A & A & $A$ & G & A & $G$ & $G$ & 49 & $4 \pm 2$ & $38 \pm 5$ \\
\hline
\end{tabular}
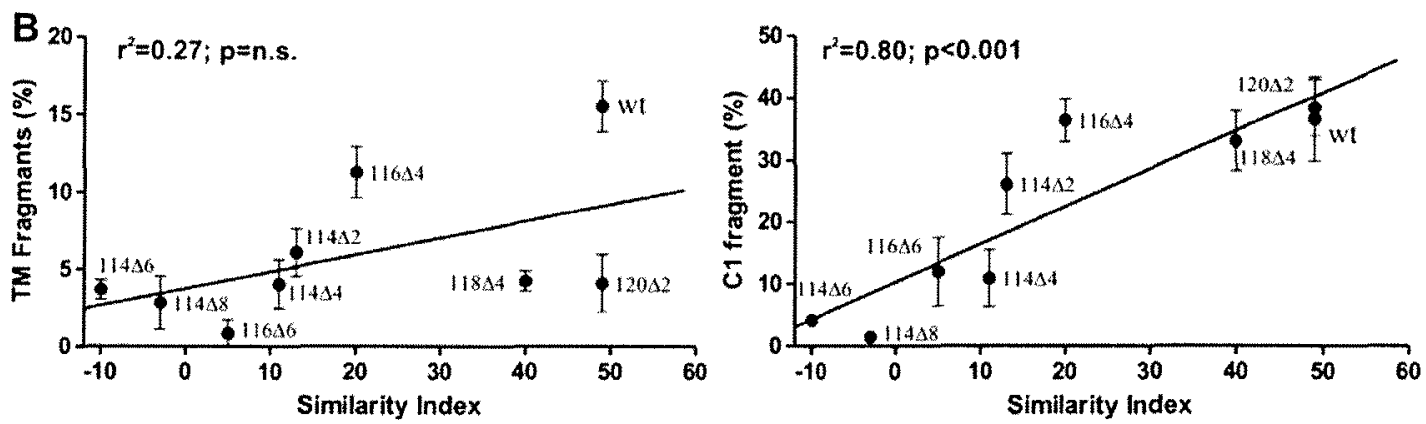

Fig. 4. Impact of the HC aminoacid sequence on $\mathrm{Pt}^{\mathrm{C}}$ topology and $\alpha$-cleavage. (A) For each $\mathrm{PrP}$ mutant the aminoacid sequence within the region of the deletion is shown. Aminoacids in boldface are identical with PrP-wt. The similarity of aminoacids $114-119$ is shown in the next column. High scores indicate high similarity to PrP-wt. The two columins on the right display the proportion of the transmembrane versions and of the $\mathrm{C} 1$ fragment. (B) In contrast to an insignificant correlation of the changes in transmembrane topology ( ${ }^{\mathrm{Ctr}} \mathrm{PrP}$ and ${ }^{\mathrm{H} m} \mathrm{PrP}$; left), $\mathrm{Cl}$-fragment abundance (rigtt) did correlate with similarity with the HC wi-sequence.

microdeletions, affecting only one specific part of the TM domain, is in accordance with previous results demonstrating that TM is subdivided in distinct topogenic entities, which regulate protein integration and orientation. In a recent study, the middle and C-terminal part of TM has been shown to counteract ${ }^{\mathrm{Ctm}} \mathrm{PrP}$ topology [15]. In contrast to the determination of PrP orientation in the ER membrane by the middle TM-segment, also $\mathrm{HC}$ plays a considerable role for topology determination. However, this segment does not influence PrP orientation; as we show here, it rather influences the integration of $\mathrm{PrP}^{\mathrm{C}}$ into the ER-membrane, as demonstrated by the marked decrease of both transmembrane forms in the various mutants.

Deletions in HC have also an impact on the generation of the $C 1$ fragment

Deletions within $\mathrm{HC}$ also exhibited notable changes in the level of an additional fragment migrating at $18-\mathrm{kDa}$. Immunoprecipitation analysis of the deletion mutants using $\mathrm{N}$ - or C-terminal PrP antibodies revealed that this fragment was $\mathrm{N}$-terminally truncated. Both, size and $\mathrm{N}$-terminal truncation were perfectly compatible with the $\mathrm{Cl}$ fragment [10]. Furthermore, the topology assay with hamster $\operatorname{Pr}^{\mathrm{C}}$ demonstrated that this fragment had lost the $3 \mathrm{~F} 4$ epitope, which is located exactly at the $\alpha$-cleavage site. All these results match the characteristics of the $\mathrm{C} 1$ fragment as defined by Autilio-Gambetti and colleagues using similar experimental approaches [10]. The analysis of transgenic mice expressing the deletion mutant PrPA114-121 in the absence of PrP-wt confirmed the impact of $\mathrm{HC}$ on $\mathrm{Pr}_{\mathrm{T}} \mathrm{P}^{\mathrm{C}} \alpha$-cleavage.

$H C$ is necessary for $P_{r} P^{C} \alpha$-cleavage

The site of $\alpha$-cleavage of $\operatorname{PrP}^{C}$ had been mapped to residues 109-111. $\alpha$-Cleavage efficiency of our mutants correlated with the similarity of the $\mathrm{HC}$ sequence between residues 114 and 119 . This stretch is part of one of the most highly conserved regions of the PrP $|16|$. Hence, the evolutionary conservation of the hydrophobic core might result from its function as a recognition site for the proteases catalyzing $\operatorname{Pr}^{c} \alpha$-cleavage, underlining the importance of this cleavage for $\mathrm{Pr}^{\mathrm{C}}$ function.

Strikingly, $\alpha$-cleavage of $\operatorname{PrP}^{\mathrm{C}}$ is mediated by the same proteases of the ADAM family that are responsible for the $\alpha$-cleavage of APP [17]. $\alpha$-Cleavage of APP prevents formation of the toxic A $\beta$ fragment and furthermore elicits various trophic stimuli. It was thus proposed that $\alpha$-cleavage of $\operatorname{PrP}^{C}$ might also have cytoprotective effects [11]. Since HC is crucial for the conversion of PrPC to $\operatorname{PrP}^{\mathrm{S}}[9,18,19], \mathrm{PrP}^{\mathrm{C}} \alpha$-cleavage destroys the region, thereby preventing the conversion process, analogous to $\alpha$-cleavage in APP. This notion is further supported by the finding that all $\mathrm{Pr}^{\mathrm{Sc}}$ fragments studied so far comprise the intact C1-cleavage site [20].

Since the in vitro topology assay only comprises cell lysate and microsomal membranes, lacking an intact plasmalemma, the generation of $\mathrm{Cl}$ fragment under these conditions substantiates the view that $\alpha$-cleavage of $\mathrm{PrP}^{\mathrm{C}}$ already occurs during the trafficking of the protein through intracellular compartments, such as ER or Golgi [21]

\section{Involvement of HC in prion pathology}

Although the evidence for a crucial role of $\operatorname{PrP}$ in some neurodegenerative diseases such as GSS or CJD is overwhelming, the exact cause for its neurotoxicity remains enigmatic. The transmembrane ${ }_{P r} \mathrm{P}^{\mathrm{C}}$ form ${ }^{\mathrm{Ctn}} \mathrm{Pr} \mathrm{P}$ was shown to be upregulated in certain prion diseases, and transgenic mice expressing high levels of ${ }^{\mathrm{Ctm}} \mathrm{PrP}$ develop neurodegeneration $[3,22]$. In addition to the function of certain topologic forms, also the $\alpha$-cleavage of PrP has been implicated 
in specific PrP function, including protection against oxidative stress [23] or against $\operatorname{Pr}^{S c}$ formation $[9,11,20]$.

Interestingly, we discovered both, an increase of ${ }^{C t m} \mathrm{PrP}$ as well as a decrease in C1-cleavage in a hereditary form of human prion disease, G114V [14], suggesting that both mechanisms, i.e. the increase of toxic PrP forms, such as ${ }^{C m} \mathrm{PrP}$, and the decrease of protective $\operatorname{PrP} \alpha$-cleavage might be relevant for $\mathrm{PrP}$ function.

Irrespective of the exact physiological role of the $\operatorname{PrP}^{C}$ and its associated pathological mechanisms we have been able, by using mutants bearing microdeletions in $\mathrm{HC}$, to further characterized this most highly conserved part of the protein as a crucial regulatory element by showing that it exerts at least two distinct functions, i.e. integration (but not orientation) of nascent $\operatorname{Pr}^{\mathrm{C}}$ polypeptides into the ER membrane and $\alpha$-cleavage of $\mathrm{PrP}^{\mathrm{C}}$.

\section{Acknowledgments}

We thank Prof. J. Collinge, MRC Prion Unit, Institute of Neurology, National Hospital, London, UK for Pmp ${ }^{-1-}$ FVB mice and Prof. W. Bodemer, German Primate Centre, Göttingen, Germany, for antibody 3B5. We thank Katharina Hüttner for expert teclmical assistance and Dr. Günter Fritz for critical reading of the manuscript. We gratefully acknowledge funding by the Deutsche Forschungsgemeinschaft through the "TransRegio-SFB 11 Konstanz-Zürich, Structure and function of membrane proteins" (TP C10) and funding by the EU Commission through the FP5 Project QLRT-2000-01924 "Strategies for the prevention and treatment of prion disease" and the FP6 Network of Excellence "NeuroPrion"/subproject PrioGen.

\section{Appendix A. Supplementary data}

Supplementary data associated with this article can be found, in the online version, at doi:10.1016/j.bbrc.2010.02.015.

\section{References}

(1) S.B. Prusiner, M.R. Scott, S.]. DeAmond, F.E. Cohen, Prion protein biology, Cell $93(1998) 337-348$.

[2] R.S. Hegde, J.A. Mastrianni, M.R. Scott, K.A. DeFea, P. Tremblay, M. Torchia, S.] DeArmond, S.B. Prusiner, V.R. Lingappa, A transmembrane form of the prion protein in neurodegenerative disease, Science 279 (1998) $827-834$.

(3) R.S. Hegde, P. Tremblay, D. Groth, S.]. DeArmond, S.B. Prusiner, V.R. Lingappa, Transmissible and genetic prion diseases share a common pathway of neurodegeneration, Nature 402 (1999) 822-826

[4] D. Falcone, D.W. Andrews, Both the 5 ' untranslated region and the sequences surrounding the start site contribute to efficient initiation of translation in vitro, Mol. Cell. Biol. 11 (1991) 2656-2664.

[5] M. Ettaiche, R. Pichot, J.P. Vincent, ]. Chabry, In vivo cytotoxicity of the prion protein fragment 106-126, J. Biol Chem. 275 (2000) 36487-36490.
[6] S.M. Zanata, M.H. Lopes, A.F. Mercadante, G.N. Haji, LB. Chiarini, R. Nomizo A.R. Freitas, A.L Cabral, K.S. Lee, M.A. Juliano, E. de Oliveira, S.G. Jachieri, A. Burlingame, L. Huang, R. Linden, R.R. Brentani, V.R. Martins, Stress-inducible protein 1 is a cell surface ligand for cellular prion that triggers neuroprotection. EMBO ]. 21 (2002) $3307-3316$

[7] EM. Norstrom, J.A. Mastrianni, The AGAAAAGA palindrome in PrP is required to gererate a productive PrPSC-PrPC complex that leads to prion propagation Biol. Chem. $280(2005) 27236-27243$

[8] T. Muramoto, M. Scott F.E. Cohen. S.B. Prusiner, Recombinant scrapie-like prion protein of 106 amino acids is soluble, Proc. Natl. Acad. Sci. USA 93 (1996) $15457-15462$

[9] C. Hölscher, H. Delius, A. Bürkle, Overexpression of nonconvertible PrPc delta114-121 in scrapie-infected mouse neuroblastoma cells leads to trarsdominant inhibition of wild-type $\operatorname{Pr} P(5 c)$ accumulation, J. Virol. 72 (1998) $1153-1159$.

[10] S.G. Chen, D.B. Teplow, P. Parchi, J.K. Teller, P. Gambetti, L. Autilio-Gambetti, Truncated forms of the human prion protein in normal brain and in prion diseases, J. Biol. Chem. 270 (1995) 19173-19180.

[11] F. Checler, B. Vincent, Alzheimer's and prion diseases: distinct pathologies, common proteolycic denominators, Trends Neurosci. 25 (2002) 616-620.

(12) F. Baumarn, M. Tolnay, C. Brabeck, J. Pahnke, U. Kloz, H.H. Niemann, M. Heikenwalder, T. Rulicke, A. Bürkle, A. Aguzzi, Lethal recessive myelin toxicity of prion protein lacking its central domain, EMBO J. 26 (2007) 538-547.

[13] S. Henikoff, J.G. Henikoff, Amino acid substitution matrices from protein blocks, Proc. Natl. Acad. Sci. USA 89 (1992) 10915-10919.

14] M.M. Rodriguez, K. Peoc'h, S. Haik, C. Bouchet, L. Vernengo, G. Manana, R. Salamano, L. Carrasco, M. Lenne, P. Beaudry, J.M. Launay, J.L. Laplanche, A novel mutation (G114V) in the prion protein gene in a family with inherited prion disease, Neurology 64 (2005) 1455-1457.

15] C.M. Ot, A. Akhavan, V.R. Lingappa, Specific features of the prion protein transmembrane domain regulate nascent chain orientation, J. Biol. Chem. 282 (2007) 11163-11171.

16] E. Rivera-Milla, B. Oidtmann, C.H. Panagiotidis, M. Baier, T. Sklaviadis, R. Hoffmann, Y. Zhou, G.P. Solis, C.A. Stuermer, E. Malaga-Trillo, Disparate evolution of prion protein donains and the distinct origin of Doppel- and evolution of prion protein donains and the distinct origin of Doppel- and
prion-related loci revealed by fish-to-mammal comparisons. FASEB J. 20 (2006) $317-319$

[17] B. Vincent, E. Paitel, F. Saftig, Y. Frobert, D. Hartmann, B. De Strooper, J. Grassi, E. Lopez-Perez, F. Checler, The disintegrins ADAM10 and TACE contribute to the constitutive and phorbol ester-regulated normal cleavage of the cellular prion protein, J. Biol. Chem. 276 (2001) 37743-37746.

18] T. Kaimann, S. Metzger, K. Kuhlmann, B. Brandt, E. Birkmann, H.D. Holtje, D. Riesner, Molecular model of an alpha-helical prion protein dimer and its monomeric subunits as derived from chemical cross-linking and molecular modeling calculations, ]. Mol. Biol. 376 (2008) 582-596.

19] D. Peretz, R.A. Williamson, Y. Matsunaga, H. Serban, C. Pinilla, R.B. Bastidas, R Rozershteyn, T.L. James, R.A. Houghten, F.E. Cohen, S.B. Prusiner, D.R. Burton, A conformational transition at the $N$ terminus of the prion protein features in formation of the scrapie isoform, J. Mol. Biol. 273 (1997) 614-622

20] A. Jimenez-Huete, P.M. Lievens, R. Vidal, P. Piccardo, B. Ghetti, F. Tagliavini, B. Frangione, F. Frelli, Endogenous proteolytic cleavage of nomal and diseaseassociated isoforms of the human prion protein in neutal and non-neural tissues, Am. J. Pathol. 153 (1998) 1561-1572.

21] H. Tveic, C. Lund, C.M. Olsen, C. Ersdal, K. Prydz, 1. Harbitz, M.A. Tranulis, Proteolytic processing of the ovine prion protein in cell cultures, Biochem. Biophys. Res. Commun. 337 (2005) 232-240.

22] R.S. Stewart, P. Piccardo, B. Ghetti, D.A. Harris, Neurodegenerative illness in transgenic mice expressing a transmembrane form of the prion protein, Neurosci. 25 (2005) 3469-3477.

23] A. Mange, F. Beranger, K. Peoc'h, T. Onodera, Y. Frobert, S. Lehmann, Alpha-and beta-cleavages of the amino-terminus of the cellular prion protein, Biol. Cell $96(2004) 125-132$. 